\title{
On Possibilities to Accelerate Engineering Application of Shape Memory Alloys
}

\author{
R.R. Ionaitis and D.B. Chernov* \\ P.O. Box 788, 101000 Moscow, Russia \\ * NIISU, 107113 Moscow, Russia
}

\begin{abstract}
It is analyzed why shape memory alloys (SMA), which could enhance reliability, safety, compactness and simplicity of technical objects are still used insufficiently. The reasons presented are as follows: lack of literature on SMA application and books on shape memory device (SMD) development; need of metallophysicist support; lack of calculation technique for high-speed SMD and representative information on heating, on influence of harmful factors. Some ways to accelerate the process of SMD implementation are proposed: application concepts; publication of explanatory dictionaries «SMA Application» or "SMD», engineering design techniques and design estimates; inter-company catalogue of standard SMD.
\end{abstract}

\section{INTRODUCTION}

There is a significant research potential in studying shape memory effect (SME), shape memory alloy (SMA) development (nickel-titanium, copper and others), SMA half-finished items (poly- and monocrystals, rods, tapes, etc.) production, shape memory device (SMD) development $/ 1 /$.

SMD are developed because of SMA unique properties: shape recovering when heated, multiple actuation, significant shift deformations, large applied forces, efficient damping, sound absorption, ductility, superelasticity, properties long-term retaining, compactness, intelligence $/ 1 /$.

First proposals on SMA use (simple, as a rule) were made by material specialists (not by designers). Later on they were replaced by numerous proposals (in the main not realised) made by professional inventors. Some SMA applications under simple conditions in terms of temperature, ambient pressure, etc. (home applications, medicine, telecommunications) were then followed by separate SMA applications in power intense equipment (space, aviation, nuclear).

Analysis of ideas and developments shows that time has come to use the SMA and SMD unique characteristics for their extensive introduction into engineering and technology.

Insufficiently quick introduction of SMD can be explained by reasons both general for any innovations and specific for SMA. General: even highly developed society can not introduce more than $1 / 10$ of what the scientists and engineers offer. Today all the room in engineering is occupied by traditional devices, that people are used to, which are well known to them and which make them feel to be specialist. Specific reasons: novelty of SMDs and lack of publications on their application.

\section{SHAPE MEMORY DEVICE POTENTIALITIES}

A pilot technology of SMA half-finished items fabrication with various geometric forms (rod, rolled stick, pressed stick, tube, wire, sheet, plate, tape, foil) were developed and mastered. Various components can be made of them in the form of rod, spiral, plate, tape, wire as well as washers, cramps. Possible deformations are strain, compression, bending, torsion; mixed.

SMA with different temperatures of transformation were obtained: well mastered by industry - up to $100-200^{\circ} \mathrm{C}$; new ones at up to $300^{\circ} \mathrm{C}$; separate ones at up to $500^{\circ} \mathrm{C}$. 
The following items are produced according to technical specifications: nickel-titanium alloys (with $\mathrm{Fe}$ or $\mathrm{Cu}$ ); copper-aluminum (with $\mathrm{Mn}$ or $\mathrm{Ni}$ or $\mathrm{Zn} . .$. ); merganese-copper (with $\mathrm{Cr}$ ).

SMDs created are different in.:

- purpose (medicine, home appliances, aerospace, aviation, gas and oil industries);

- design of devices (drives, thermomechanical joints, emergency systems);

- number of actuation cycles (single, multiple, multicyclic);

- speed of action (low-, middle-, highspeed);

- way of memory effect initiation (by heating, irradiation, pressures);

- way of energy transfer or initiation (directly to an object, via a drive or an electric circuit);

- SMA-element design (spiral springs, rods of various profiles, washers, torsion elements)

- way of kinematic initial shape recovering (by biasing body, two-way SME, forced recovering);

- way of SMA element material recovering the initial phase condition;

- size (many-metre buildings, middle sizes, micro).

Many SMA and SMD are patented. Information on SMD can be found in books, catalogues, conference proceedings. However, no great demand is observed since SMA-designs created earlier were developed for specified, as a rule, narrow problems.

A big array of SMAs with their parameters related to recovery degree, forces actuation, temperatures and others allows to consider possibilities of SMD wide application in engineering and technology.

\section{VITAL CHARACTER OF SMA AND SMD UP-TO-DATE APPLICATION}

Any and power intense equipment, in particular, are characterised by:

- high potential danger;

- great variety of problems to be solved;

- incomplete raw data, lack of complete information on processes running in structures under creation;

- need to co-ordinate efforts of different specialists;

- short terms for work implementation.

Operating conditions of technical facilities at power plants are very complicated due to large variety of ambients; coolant aggressive; high operating parameters; influence of harmful production factors.

Therefore it is vital in up-to-date engineering :

- to increase reliability and safety (using a number of technical methods);

- to make devices compact (with permissible power intensity);

- to achieve simplicity of designs (and modularity of their construction);

- to reduce effect of harmful factors (irradiation, etc.).

Reliability and safety of modern installations is estimated, ensured, increased by way of gradual and complex application of reliability and safety principles and technical criteria such as: inherent safety, automated actuation, fast action, safe failure, redundancy, independence, variety, protection in depth, controllability, tested state, passivity, modularity, simplicity, harmless operation, complete actions $/ 2 /$.

SMA and SMD are able to solve the above technical problems on account of:

- principle of variety, i.e. use of devices based on unconventional principle of action;

- high forces applied, strains, compact designs and other particularities;

- complex functionality, i.e. realisation of check, implementation and other functions in one device;

- high intelligence, i.e. direct actuation by an operating parameter.

\section{SMA AND SMD APPLICATION PROBLEMS}

\subsection{Positive factors}

The factors producing positive effect on the problem of SMA application are as follows:

- significant research potential in SMA development and study, 
- production of various materials in terms of grades and properties in a wide range,

- various methods and techniques for shape memory parameters determination,

- advanced approach to designing thermomechanical connecting devices,

- convincing examples of successful SMD use in various areas of technology and medicine.

\subsection{Negative factors}

The major problem of SMDs engineering application is their substantial novelty, they have little in common with their analogues, lack of prototypes and small (with regard to the whole scope of technology) experience in development activity. Other negative factors of such narrow SMD introduction into engineering and technology are the following:

- a substantial and still getting larger gap between theory and practice,

- lack of rigorous concepts on SMD application in various branches of engineering and technology,

- wide spread of SMA and SMD information over numerous places difficult to access,

- lack of systematised information on patented designs and structures under production,

- lack of general (unified) or standard techniques (and terminology) of thermomechanical parameters determination at an interregion level,

- lack of comparative characteristics of commercial SMA materials and their properties,

- lack of standard calculation and designing techniques,

- lack of engineering manuals on material properties, test techniques, design methods and examples of design developments.

\subsection{Patents application}

Information on patents, available designs and type decisions is of great help at the initial stage of designing. There are thousands of patents on SMA and SMD. However, patent search in this area is difficult due to the following reasons:

- patents on the given subject are not splitted into classes,

- SMA and SMD are spread over many different classes,

- patent descriptions do not incorporate keywords,

- shape memory is not mentioned in patent name.

As a result, automatic search efficiency is close to zero. Due to the said reasons patent search is efficient only if it is made by a specialist in the field of SMA and SMD application.

Presently we do not even know exactly the total number of patents on SMA and their application. No one country performs a global-scale patent search due to organisational and economic reasons, in particular, a limited number of specialists in this field of knowledge and difficulties of their part-time involvement to this activity. Some personal «patent purity» searches were only made for subsequent patenting of a concrete design. Serious patent and engineering studies on SMD are not available.

\subsection{Choice of alloys}

The matter of SMA choosing is not satisfactory either. At present, several types of NiTi- and $\mathrm{Cu}$ - basedSMA are produced in the form of various half-finished items. Well-outlined criteria for SMA evaluation and comparison are necessary for a justified choice of a SMA design. However, a set of specified parameters, their notation, terminology and techniques for SMA testing are not consistent. This does not allow to understand and interpret identical technical parameters and characteristics (temperature, strain, energy and fatigue) in a synonymous way. In addition to a shape memory parameters, designers need a large scope of SMA properties. To evaluate the available thermomechanical parameters correctly they should also be compared in terms of their accuracy and trust worthiness. For this, test techniques for each group of parameters should also be systemised and unified. 


\section{PRIORITY OF SMD DEVELOPMENTS}

The problems stated can and will be solved quickly and efficiently if efforts of SMD designers the number of which is presently not large, are concentrated upon the most important directions in SMA and SMD application governed by technical problems and SMD potentialities.

\subsection{Concept}

SMA development is, in the first place, intended to provide:

- reliability and safety, $\bullet$ realisation of the variety principle, $\bullet$ compact design, simplicity, modularity,

- high intelligence and complex functionality, $\bullet$ reduction in harmful factors effect.

SMD are first proposed for use as additional (rather than alternative) means for existing devices. In future, during highly qualified design work unique characteristics of SMA will allow SMD to become traditional and to efficiently use them for replacement of (rather then adding to) existing structures.

SMD creation is recognised vital:

- to maintain reliable operation i.e. for providing joints, leak tightness, strengthening in the form of pipe plugs (end and lateral), seais (flange gaskets, valve saddles), thermal clutches, tires, fixating, welds strengtheners;

- to shutdown basic process as power rise indicators, transforming controls elements, direct actuators;

- to control fluid ambient flow rate by self-locking thermal devices and controlled valves;

- to repair and mount in the form of special contrivances, process equipment small-dimension manual tools;

- to provide fire safety in the form of fire valves, etc.

The lack of a direct emergency signal for temperature related to mode parameters in many technical facilities (as a rule, coolant temperature, gas pressure and other parameters are available) constitute serious difficulties for SMDs as temperature-responding devices.

Required characteristics of SMD as used in power intense equipment: compact design; control of actuation temperature; high actuation speed; full or partial (but sufficient for operation) preservation of SMD required properties under harmful factors effect (irradiation and others.). Possible ways of application: use them out of areas with a strong influence of operating parameters; distant transmission of emergency signal (sensitive and executive elements separation); easy replacement (for short life).

The following functions are to be performed as SMDs are used in safety means: facility control and emergency signals formation, transformation, amplification and performance (realisation of protective action); diagnostics (readiness of SMD to operate and the fact of its actuation).

\subsection{SMD use for mounting and repair}

High-quality and reliable SMDs used for mounting and repair can increase equipment service life, reduce costs of potential repair and dose commitments governed by mounting rate. Simple technology for SMD joints mounting and assembly enables to abandon expensive technologies, high temperature heating of materials, inspection of welded joints quality and to reliably (in a strong and leaktight manner) join diverse (impossible to be welded together) materials.

Thermomechanical joints, seals, fixing locks for pipe lines and valves, fastening SMD-joints seem to have future. Internal thermal couplings, fixing locks, strengtheners are especially vital for a number of technical installations, because access from outside is very often impossible.

Application of small-size jacks, presses, tube benders, removers, scissors, flatteners and other power SMD equipment for mounting and repair may substantially reduce the time for work implementation, related to limited room in power intense installations, reduce their cost, reduce dose commitments, ensure easy transfer.

Due to SMD high load-bearing features they enable to raise cost-efficiency of load carrying devices compared with other devices based on other principles of action. 
Plates, cylinders, tubes, rods can be used in power SMD-drives as load-carrying SMD components working for extension, compression, bending. Heating can be performed by gas blowing, coolant pumping or by electric heaters of known or principally new types, by direct ohmic heating (in case of SMD wire). Return action can be realised by cooling of basic components, heating counter-components, by common revocable springs, components with elastic shape memory (superelastic).

\subsection{SMD application in safety means}

SMDs are to possess the following properties as used in safety means (SM): small delay in actuation; free introducing into the facility active zone; high speed of introduction; high efficiency of process controi; sufficient deceleration at the end of movement; acceptable efforts when recovering initial condition.

Typical SM components based on SMA must ensure: reception and transfer of emergency signal; retention and introduction of a control member; transformation of its geometric form and deceleration.

Safety (self-actuating) SMDs (control and executive) should be actuated directly by an operating parameter: power size, deterioration of heat removal, changed pressure or coolant flow.

Self-actuating SMD devices are efficient when control substances in fluid (liquid, gas, fluid metal) or loose (sand) state are directly introduced into the active zone.

\subsection{Pipeline stop- devices based on SMA}

Stop (S) devices with various executive mechanisms (actuators) are widely used in hardware.

SMD actuators are worth-while developing to reduce overall dimensions and mass; eliminate dry (metallic) friction pairs; ensure separate elements redundancy; expand the modularity of designs.

Self-stopping SMDs are possible to be used: for excluding (limiting) outflow; in case of tube rupture; as ambient temperature changes; fixing and decoupling of operating members.

Controlled S SMDs are possible to be of built-in, combined and external modifications. When loadcarrying components are located inside, the structure is very compact, but it requires SMAs with actuation temperatures, exceeding the coolant temperature. Besides, inside the body there are many details giving rise to resistance and requiring their reliable fixing in liquid flow.

There can be SSMD with external load carrying elements and with a system transferring motion to stop members located inside. The most prospective devices are straight-flowing SDs of which the basic element is an elastic element of a hose type. This element is clenched in its central part by one or two SMD-drives with clenches, load-carrying elements and a heater. The elastic element opens the flowthrough part by means of the coolant pressure.

SMD actuators are prospective for enhancing operation reliability, compactness and simplicity of structure.

\section{FURTHER WORK PROCEDURE}

The following sequence of work is proposed for the first stage justification on SMD application ii engineering and technology.

6.1. SMD patent and technical studies (since 1970, for 7 developed countries): application in power intense equipment; use for mounting and repair, pipelines stop devices with transformed flow-through part, self-stopping devices, power actuators (drives), executive operating members; straight-acting actuators.

6.2. Marketing Bases on SMA application in engineering and technology (collection and analysis of information from plants and laboratories in different countries): alloys composition (structure), alloying elements, indicative parameters of shape memory (actuation temperatures, forces, strains), processing and alloying effect, forms of half-finished items (blanks): round, flat, plates, rods, tubes, tapes, wire, etc.; 
properties distribution over cross section, mono- and polycrystals, technical specifications for alloys, devices, test methods, cost information and estimates (melting, rolling, forging, giving certain properties, testing, certification, etc.); researchers, designers, producers.

6.3. Generalisation of data on SMD use in engineering and technology. Analysis of home and medical applications for potential use in equipment: preparation of an information note and analysis of catalogues of world-known companies -- producers of SMA and SMD, proposals on making of an inter-company world-wide catalogue; working out requirements to parameters and features of SMD as using them in power intense equipment; analysis of ways to be applied for heating and cooling; analysis of ways for ensuring high-speed actuation with different types of heating, analysis of ways and devices for protecting against harmful factors.

6.4. Development of recommendations on $\mathrm{SMD}$ use in engineering: for mounting and repair, maintaining normal operation, under emergency trip (shutdown), for coolant flow rate control, for safety, etc.

\section{CONCLUSIONS}

1. Unconventional technical means including shape memory devices are expedient to be used in engineering and technology especially power intense, for enhancing reliability and safety level of power plants (by using the principle of diversity), to raise product compactness, reduce personnel irradiation, etc.

2. At present there is a certain research potential in SMD use: application strategy, pilot technology for half-finished items fabrication, a set of alloys with different actuation temperature, principle and design decisions, theoretical base, experimental base for studies, experimental characteristics, inspection and operating experience (partially under in-pile conditions), SMD optimisation program in terms of higher stability to irradiation, fast- acting, corrosion stability.

3. Out of all the variety of potential SMDs prior use is offered to:

- motionless thermomechanical joints, seals, plugs, fixing locks, etc.;

- self-actuating two-position emergency devices designed to control plant basic process and ambient flow rate;

- electrically operated actuators to be used in stop valves, and small-dimension tooks.

4. Initially SMD are offered as an addition (as redundancy, higher safety margin) to existing devices. Efforts are given to qualified SMD designing, aiming at their conversion to traditional devices.

5. To accelerate the process of SMD introduction to nuclear engineering and technology it is necessary to:

- make an inter-company catalogue of SMD currently available,

- develop an explanatory dictionary "Shape Memory Devices" (or "Shape Memory Alloys Application"),

- set up strict distinction study and development stages, provide SMA and SMD with technical requirements, between certification, etc. to separate them from researchers and developers;

- develop engineering techniques for SMD design and calculation including ways of quick heating (and cooling), fast response calculations, operation in aggressive media.

\section{References}

[1] International Meetings Proceedings: SMST-94; SMA for Power Systems (USA, 1994); ICOMAT-95; Actual Problems of Toughness, Russia, St-Petersburg, 1995-96; Intelligent Materials 1996; Actuator 96; SMST-97.

[2] Ionaitis R.R. Complex of Criteria to Determine, Ensure and Increase Safety Level of Power Plants. Russia «Atomic Energy», V. 79, № 2, 1995, p. 83-88. 